\title{
Inovação e internacionalização bioindustrial em Minas Gerais 2001-2004: como potencializar oportunidades e avançar rumo a 2020?*
}

Valéria M. M. Judice**

Sandro Márcio da Silva***

Adelaide M.C. Baeta ****

\section{Resumo}

Visando à formulação de políticas de desenvolvimento bioindustrial em Minas Gerais, 0 artigo analisa e integra resultados de duas pesquisas realizadas em 2004: uma delas, empírica, sobre a evolução bioindustrial mineira no período 2001-04 e a outra, um estudo de políticas bioindustriais internacionais. Duas constatações da primeira pesquisa são percebidas como oportunidades a serem promovidas, à luz de modelos de políticas internacionais, identificados na segunda pesquisa. Numa amostra de 42 empresas bioindustriais, selecionadas de 75 em operação naquele estado, fica evidente, de um lado, um decréscimo no número de empresas, mas também uma ampliação da capacidade inovadora, indicada pelo surgimento de novos entrantes com maior potencial tecnológico, produção de nível mundial e investimentos venture capital. De outro lado, com relação à internacionalização, constata-se, nas organizações pesquisadas, um incremento das exportações, da intenção de "exportar mais no futuro" e avanços quanto à capacidade de atuação no mercado externo. Após a avaliação e o detalhamento dessas oportunidades, ao final, são propostas políticas para promoção e apoio ao setor bioindustrial, conforme políticas-padrão praticadas em nível internacional.

Palavras- chave: biotecnologia, internacionalização, inovação, políticas bioindustriais, Brasil, Minas Gerais.

\begin{abstract}
This paper seeks to contribute to the biondustry policy-making process in Brazil. It analyzes and integrates results of two projects carried out in 2004: an updating study of the bioindustrial evolution in Minas Gerais State during the period of 2001-04 and a survey on international bioindustrial policy. Two empirical results of the first study are reviewed as potential opportunities for application of standard international policy instruments, as observed in the survey. The investigation considered a sample of 42 biotechnology companies out of a group of 75 operational companies in the state of Minas Gerais. The research shows an evolution from 2001 until 2004 that includes a slight decrease in the number of companies. At the same time, it shows the upsurge of new entrant companies with higher technological potential, innovative capacity, international standard products and venture capital investments. In terms of internationalization, increased biondustrial exports were found as well as "intention to export more in the future" and enhanced abilities to sell abroad. The article summarizes such opportunities and suggests instruments for supporting public policy aiming to promote the international competitiveness of the Brazilian bioindustry .
\end{abstract}

Key words: biotechnology; internationalization; innovation; bioindustrial policy; Brazil; Minas Gerais.

\footnotetext{
* Uma versão preliminar deste artigo foi apresentada no XXIII Simpósio de Gestão de Inovação Tecnológica, USP- PGTEC, em Curitiba, 19-22 out. 2004.

** Vice-coordenadora, professora e pesquisadora do mestrado profissional em administração (MPA) da Fundação Pedro Leopoldo (MG). E-mail: vjudice@ uol.com.br. Endereço: Caixa Postal 123 / Cep:33.600-000 Pedro Leopoldo-MG

*** Professor e pesquisador do mestrado profissional em administração (MPA) da Pontifícia Universidade Católica de Minas Gerais (PUC-MG). E-mail: sandromarcio@ pucminas.br. Endereço: Av. Dom José Gaspar, 500 - Coração Eucarístico / CEP:30535-610 Belo Horizonte-MG

**** Coordenadora, professora e pesquisadora do mestrado profissional em Administração (MPA) da Fundação Pedro Leopoldo (MG). E-mail: Adelaide@ task.com.br. Endereço: Caixa Postal 123 / Cep:33.600-000 Pedro Leopoldo-MG

Artigo aceito para publicação em novembro de 2004 e aceito em março de 2005.
} 


\section{Introdução}

Das diversas tecnologias emergentes, a biotecnologia é uma área promissora. Com os recentes avanços em genômica, proteômica e bioinformática, a bioindústria vem contribuindo para o crescimento econômico e social trazendo novas soluções para problemas relativos à saúde humana e animal, ao agrobusiness, ao meio ambiente e à criação de novos materiais industriais.

Alguns exemplos de avanços efetivos e potenciais são as plantas que poderão ser utilizadas como vacinas, os vegetais enriquecidos com vitaminas, os novos medicamentos produzidos por engenharia genética, os órgãos e tecidos para transplante sem problemas de rejeição, os microrganismos geneticamente modificados para biorremediação (despoluição) e os biomateriais de aplicação em saúde humana (nanomedicina) e de uso industrial (polímeros, produtos químicos e de eletrônica molecular).

No âmbito internacional, a inovação bioindustrial já apresenta muitos resultados de sucesso e comercialização. Conforme a BIO, organização da bioindústria nos EUA, em seus 30 anos de atuação mundial, o setor já produziu mais de 100 medicamentos e ainda tem outros 350 produtos em testes clínicos. Seu valor para a sociedade se consolida, estimando-se que responda por mais de 1/3 do PIB mundial (SIMON e KOTLER, 2004).

No Brasil, estudo identificando e avaliando o desenvolvimento de empresas de biotecnologia constatou em 2001, a existência de 304 empresas em operação, concentradas particularmente em Minas Gerais (29\%) e São Paulo (43\%) (BIOMINAS, 2001).

Em Minas Gerais, diversos fatores de ordem histórica favoreceram a concentração geográfica da bioindústria, como a tradição e a competência das escolas de medicina, bioquímica e farmácia; algumas delas, entre as primeiras do Brasil (MARCOLIN, 2004). Além de manifestações de expertise e capacitação das universidades e centros de pesquisa, devem ser consideradas iniciativas que revelam uma visão local empreendedora e pioneira em relação a nichos de mercado para produtos e serviços voltados para a saúde humana. Esse conjunto de fatores consolidou um ambiente que estimula os negócios, contribuindo para o surgimento de várias micro, pequenas e médias empresas concentradas na Região Metropolitana de Belo Horizonte.

Um exemplo típico desse desenvolvimento foi o surgimento, em 1976, da primeira empresa de biotecnologia brasileira, a Biobras, um spin-off universitário e bem-sucedido exemplo do pioneirismo de Minas Gerais numa atividade industrial de base científica. Destaca-se também a iniciativa de sete empresas do setor que em 1990 criaram a Fundação Biominas, uma entidade tecnológica de suporte, catalisadora de ações de integração, difusão de conhecimento e de governança do setor.

Em 1990, cerca de 10 empresas de biotecnologia estavam em operação em Minas Gerais. Em 2004, o estado já concentrava mais de 80 empresas, incluindo corporações de grande porte, nacionais e internacionais, como Monsanto e Syngenta (no Triângulo Mineiro), Novo Nordisk e Vallée (Montes Claros) e Labtest e Diamed (Região Metropolitana de Belo Horizonte).

Em 1999, um estudo da McKinsey para a Federação das Indústrias de Minas Gerais (Fiemg) confirmou a existência de um "embrião de cluster" em biotecnologia na região de Belo Horizonte (Fiemg, 2000). Esse estudo e a atuação promotora da federação das indústrias tiveram um papel mobilizador na primeira aproximação entre os diversos atores econômicos, sociais e políticos regionais, os quais, naquele momento, viram-se engajados na transformação do potencial cluster em ação de desenvolvimento regional.

Há um reconhecimento público e acadêmico da existência de um "arranjo produtivo local" (APL), particularmente na região da capital e cidades vizinhas, num raio de $100 \mathrm{~km}$, o que é confirmado por diversos estudos (CASSIOLATO e LASTRES, 2000; LEMOS, 2000; FIEMG, 2000; FAJNZYLBER, 2001; BIOMINAS, 2001; JUDICE e BAÊTA, 2002; JUDICE e BAÊTA, 2003; SOUZA, 2002). 
Ações de promoção e suporte a APLs de biotecnologia vêm se desenvolvendo até hoje. No caso da bioindústria em Belo Horizonte, além do apoio do sistema Fiemg, Instituto Euvaldo Lodi (IEL-MG) e do Sebrae, chama atenção a atuação da Universidade Federal de Minas Gerais (UFMG) e o apoio dos governos estadual e municipal, através da Secretaria Estadual de Ciência e Tecnologia e da Prefeitura de Belo Horizonte. Iniciativas de destaque da parte dessas duas últimas instituições são, de um lado, a construção de uma bioincubadora (1997) e, de outro, a recente implementação do projeto Parque Tecnológico de Belo Horizonte, que deverá abrigar muitas das bioempresas hoje em processo de incubação ou de pré-incubação.

Apesar desses esforços para promover e apoiar o desenvolvimento do APL de biotecnologia, em um estudo para atualização de informações sobre a bioindústria em Minas Gerais no período 2001-04 (JUDICE e SOARES, 2004) observa-se um decréscimo de 89 empresas identificadas em 2001 (BIOMINAS, 2001) para 83 (75 operantes e 8 em constituição) em 2004, representando um crescimento negativo de $-6,7 \%$ (ou de $-15,8 \%$, considerando apenas empresas em funcionamento).

Embora esse decréscimo possa ser relativizado, conforme será discutido adiante, o índice negativo evidencia a necessidade de políticas de promoção e apoio à biotecnologia, por sua importância estratégica para Minas Gerais e para todo o país; sobretudo, se considerarmos que num período recente (2000-04), a bioindústria internacional vem sendo sistematicamente apoiada através de programas de suporte, pelos altos investimentos e pela concessão de incentivos e créditos fiscais (JUDICE, 2004).

Quanto ao decréscimo do número de empresas, cabe destacar que a pesquisa de Judice e Soares (2004) mostra a criação de 20 novas empresas no referido período, indicando vitalidade no setor. O saldo negativo resulta da retirada de 26 empresas da base de dados original, seja por reclassificação - tendo em vista padrões internacionais de comparação ${ }^{1}$ - seja pelas fusões e aquisições no período ou por mudanças de setor, cidade e estado. Outra razão para o saldo negativo seria a "mortalidade infantil" de empresas identificadas como nascentes na pesquisa de 2001, as quais não sobreviveram a dificuldades de ordem macroeconômica nos anos 2001-04, ou então, que sobreviveram precariamente, entrando em "hibernação" e se posicionando em stand by, isto é, na expectativa de um cenário econômico mais favorável.

Se a fluidez da evolução do parque bioindustrial mineiro no período 2001-04 está dentro dos padrões de um setor emergente e se apresenta coerente com o cenário de baixo crescimento econômico do Brasil naqueles anos (BADIN, 2003), pouco se sabe sobre a natureza da evolução interna das empresas no mesmo período. Também pouco se sabe de suas interações e colaborações com outras empresas, universidades e com o ambiente internacional, no que diz respeito a processos de inovação, integração interorganizacional e internacionalização no período em questão.

A pesquisa empírica parte desse ponto para avaliar a evolução bioindustrial em Minas Gerais no período destacado, considerando os arranjos produtivos locais para a região de Belo Horizonte e buscando identificar as mudanças no cenário estadual. Em particular, este artigo se debruça sobre dois aspectos principais: os processos de internacionalização e capacitação para os mercados globais e a capacidade/potencial de inovação nos segmentos bioindustriais observados.

Para contextualizar a bioindústria no mundo, é feita uma breve abordagem sobre políticas e iniciativas internacionais de desenvolvimento do setor, a partir de 2000-04, tendo por base um recente estudo (JUDICE, 2004), visando com isso proporcionar uma referência à elaboração de políticas de desenvolvimento bioindustrial no Brasil.

Empresas fornecedoras de equipamentos, de consultoria e públicas foram retiradas da base de dados originais, por não serem computadas em 2 estudos recentes adotados como base para o presente estudo (TRAORÉ, 2003; US DEPARTMENT OF COMMERCE, 2003). 


\section{Referencial teórico}

Três aspectos sustentam o quadro teórico para a elaboração deste artigo. Em primeiro lugar, observa-se a importância da abordagem ou "moldura" dos arranjos produtivos locais (APLs) e/ou clusters para o desenvolvimento de um setor emergente como a bioindústria. Esse enfoque é sustentado empiricamente por múltiplas e diversificadas experiências internacionais no setor, algumas das quais são aqui delineadas em seu atual formato. Em segundo lugar, busca-se uma integração dos temas "arranjo produtivo local", "internacionalização" e "inovação tecnológica", cujo fio condutor é sua atual contextualização como política industrial, especialmente no Brasil. Em terceiro, são apresentados, brevemente, alguns elementos dos atuais modelos internacionais de desenvolvimento bioindustrial, em particular no que concerne à recente escalada (período 2000-04) de políticas públicas, iniciativas de apoio e promoção bioindustrial governamentais e privadas, levadas a efeito, especialmente, em países líderes em biotecnologia (EUA, Reino Unido, Canadá, Alemanha, Israel e Cingapura).

\subsection{0 enfoque de arranjos produtivos em biotecnologia}

Arranjos produtivos locais (APLs), aglomerados produtivos ou clusters ${ }^{2}$ têm sido considerados o formato organizacional característico da biotecnologia, pela sua natureza emergente e sua base inovadora, fundamentada em avanços científicos. Isso implica a necessidade de manter proximidade com instituições que são fontes de conhecimento, como as universidades e os centros de pesquisa. Muito mais do que a competitividade (PORTER, 1999a; PORTER, 1999b), a literatura sobre APLs em biotecnologia ressalta como peculiaridade de tais arranjos a importância de compartilhar o conhecimento num setor industrial sciencedriven (COOKE, 2001; COOKE, 2003; POWEL et al, 2002; TALLMAN, 2004), o estágio nascente e fluido do setor (FELDMAN; RONZIO, 2001) e a relevância das colaborações e alianças interorganizacionais durante o ciclo de aprendizagem das empresas (OLIVER, 2001; WEISENFELD, REEVES e HUNCK-MEISWINKEL, 2001; ZOLLO, REUER e SINGH, 2002).

No âmbito internacional, as empresas de biotecnologia têm se desenvolvido no formato de clusters ou arranjos produtivos, como demonstram inúmeros exemplos. Recentemente, nos EUA, 12 clusters foram destacados (DE VOL et al, 2004), como as bem-sucedidas (e que ainda causam impacto) experiências pioneiras em San Diego e San Francisco (Califórnia), os desenvolvimentos em Boston e na Carolina do Norte, (AUDRETSCH, 2001) e experiências mais recentes e institucionalmente implantadas de "clusterização" em áreas de Washington (FELDMAN e FRANCIS, 2003).

No Reino Unido, além da importância de Cambridge, na Inglaterra (COOKE, 2001; COOKE, 2003; CASPER e KARAMANOS, 2003), cinco áreas de concentração são apontadas na literatura especializada: Oxford, Surrey, Sussex, Kent e a Escócia (SAINSBURY OF TURVILLE, 1999). Na Alemanha, as regiões de Munique, Colônia e Heidelberg são as mais importantes e conhecidas (KAISER, 2003). No Canadá, Montreal e Toronto são os principais centros (NIOSI e BAS, 2003); e em Israel, um país territorialmente pequeno, cinco aglomerações universitárias criam um ambiente de estímulo à expansão da bioindústria (KAUFMANN et al, 2003). Finalmente, também são observadas concentrações em diversos países em desenvolvimento, como Malásia (KANDIAH, 2003), Coréia (SANG, 2003) e Índia (FELLER, 2003). ${ }^{3}$

No Brasil, estudos recentes têm ressaltado a importância dos arranjos produtivos locais, seja como ambiente de inserção e superação de barreiras tecnológicas, financeiras e de escala para o crescimento de micro, pequenas e médias empresas (LASTRES, CASSIOLATO e MACIEL, 2003), ou como conjuntos de redes interorganizacionais estruturadas com reais possibilidades de implementar uma política industrial no país (FLEURY e FLEURY, 2004).

\footnotetext{
2 A denominação cluster tem sido mais utilizada nas abordagens de origem anglo-saxã, conforme observado por Cassiolato e Lastres (2003).

3 São poucas as concentrações bioindustriais na América Latina, embora se identifiquem países de maior destaque como Brasil, Cuba, Costa Rica e México.
} 
De diferentes perspectivas, o APL de biotecnologia mineiro vem sendo analisado em sua evolução, desde os primeiros estudos de reconhecimento e identificação (LEMOS, 2000) CASSIOLATO e LASTRES, 2000; FIEMG, 2000) até enfoques mais recentes, tratando de sua viabilidade econômica, fatores de inovação, competitividade, desenvolvimento regional, financiamento e investimentos (BIOMINAS, 2001; FAJNZYLBER, 2001; JUDICE e BAÊTA, 2002; JUDICE e BAÊTA, 2003; SOUZA, 2002).

\subsection{Arranjos produtivos, internacionalização e inovação em biotecnologia}

Historicamente, a política e as práticas industriais brasileiras (e da América Latina) têm enfatizado muito mais a chamada "substituição de importações" do que propriamente promovido a exportação. Uma das razões disso é atribuída à prevalência de um "pessimismo exportador", da idéia de que o acesso dos produtos brasileiros ao mercado internacional é limitado e assim permanecerá (FURTADO, 2004). Nesse sentido, em termos de competitividade, a comercialização e marketing de produtos tem sido apontada como uma das grandes deficiências das empresas brasileiras no mercado internacional (FURTADO, 2004). Em relação ao quadro mais recente das exportações do Brasil, o que merece atenção não é o volume de produtos exportados em si, mas o fato de serem produtos de médio e baixo valor agregado.

Recentemente, criou-se a expectativa de que arranjos produtivos locais possam ser mecanismos efetivos para superar essas dificuldades no processo de internacionalização das empresas brasileiras. As análises sobre a construção e a consolidação de APLs no Brasil vêm, sobretudo, enfatizando seu potencial de inserção na economia globalizada e o papel dessas redes de micro, pequenas e médias empresas efetivamente organizadas e integradas como agentes de implementação de política industrial (SCATOLIN et al, 2003; SOUZA et al, 2003; FLEURY e FLEURY, 2004).

È necessário observar que apesar do grande esforço feito ultimamente para elaboração de políticas de desenvolvimento no Brasil com foco em arranjos produtivos, a mera disseminação do modelo de arranjos produtivos ou aglomerados locais não cria as condições necessárias para o desenvolvimento. Em particular, no caso de APLs de base tecnológica, tem ficado evidente que às limitações tecnológicas de determinada região se somam outras limitações presentes no ambiente socioeconômico local, regional e nacional, restringindo o pleno desenvolvimento de iniciativas que visam à geração de tecnologia. Trata-se de limitações como:

- a relativa baixa disponibilidade de capital de risco (em comparação com os padrões internacionais),

- a falta de apoio à propriedade intelectual e à negociação tecnológica,

- o incipiente empreendedorismo e o impacto econômico que barreiras culturais à formação e à ampliação do capital social exercem sobre a produtividade, contribuindo para a elevação dos custos; e

- os empecilhos burocráticos.

Por outro lado, diversas pesquisas (OCDE, 2001; OLIVEIRA JUNIOR e CYRINO, 2002; BADIN, 2003) vêm analisando as efetivas potencialidades de internacionalização de empresas e setores industriais brasileiros em diferentes etapas, onde progressivos "graus de internacionalização" são alcançados:

- etapa primária, com exportação direta (ou através de terceiros);

- escritórios de comercialização;

- alianças estratégicas com empresas estrangeiras; e

- instalação de subsidiárias ou unidades de produção próprias (OLIVEIRA JUNIOR e CYRINO, 2002).

Em relação à biotecnologia, particularmente quanto aos estágios de desenvolvimento da internacionalização, é possível observar um ciclo mais longo de etapas de amadurecimento, envolvendo, em especial:

- o atendimento à regulamentações específicas do setor,

- a criação de competências tecnológicas e de inovação; 
- e, sobretudo, o desenvolvimento de competências internas de gestão e internalização de técnicas avançadas de organização empresarial e marketing, além da criação e consolidação de boa reputação e de marcas de qualidade.

No que diz respeito à comercialização e marketing, o mercado mundial de biotecnologia vive um momento de inflexão, em que o predomínio da estratégia de mercado de massa começa a ser questionado pelo novo paradigma da medicina personalizada. A construção de biomarcas globais está cada vez mais associada à personalização, à capacidade de criar condições de medicina e biofarmacêutica personalizadas e de alta qualidade.

As próximas etapas de desenvolvimento da pesquisa pós-genômica e as tecnologias de informação tendem a fazer com os resultados das pesquisas sejam obtidos cada vez mais rapidamente, exigindo abordagens de marketing totalmente diferentes em relação aos produtos das "ciências da vida" (SIMON e KOTLER, 2004), tanto no Brasil quanto no exterior. Nesse sentido, observa-se, em empresas de Minas Gerais, mudanças que expressam um significativo movimentos rumo a exportações e ao estabelecimento de condições de regulamentação e aceitação de produtos no mercado externo.

\subsection{Políticas estratégicas internacionais em bioindústria - apoio prioritário e ações rumo a 2020}

Um estudo exploratório de experiências internacionais de desenvolvimento da bioindústria (JUDICE, 2004) evidenciou que, nos últimos anos (2000-04), vêm ocorrendo, em diversos países líderes nesse setor, ações sistemáticas e implantação efetiva de políticas de incentivo à bioindústria, com projeção de alto crescimento para os próximos 10-15 anos. Basicamente, existem no mundo dois "modelos" de políticas de desenvolvimento bioindustrial, e o contraste entre esses modelos traz importantes lições para o Brasil.

O "modelo 1", que poderia ser chamado de "modelo fortemente indutor e estruturador da organização bioindustrial" está institucionalizado em países como os EUA, Reino Unido, Canadá, Alemanha, Israel e Cingapura. Vem se impondo mais fortemente nos anos 2000, com perspectivas e propostas de um novo ciclo de investimentos públicos e privados. Ele estabelece uma passagem ou ruptura tecnológica da fase de "instalação" da tecnologia para uma etapa de amadurecimento tecnológico, como aperfeiçoamento (deployment) e aposta consistente na tecnologia (PEREZ, 2003).

Acredita-se que essa passagem crítica se estabeleça com os avanços da pesquisa genômica e proteômica (e demais "ômicas") e seu potencial comercial numa etapa de expansão da medicina personalizada. Do ponto de vista das políticas e investimentos públicos e privados, um dos principais fatos reveladores da atual conjuntura de amadurecimento biondustrial é o posicionamento estratégico dos países que se colocam proativos, através de pesadas apostas e induções estruturadoras do setor.

O "modelo" 2 (se tal política pode ser chamada de "modelo") seria definido como "fracamente indutor e de baixo nível de organização bioindustrial". Apresenta reduzido nível de indução, organização e estruturação de políticas de suporte e disponibilidade de informações e de conhecimento. $\mathrm{O}$ único caso internacional estudado foi o da Espanha, que adota políticas indistintas para a biotecnologia; isto é, inseridas na política mais geral de pesquisa e desenvolvimento e inovação, sem especificidade.

$\mathrm{Na}$ implantação do modelo 1 é freqüente se recorrer a cinco instrumentos políticos básicos:

- apoio ao desenvolvimento de arranjos produtivos ou clusters espontaneamente existentes ou induzir a sua criação;

- implantação de infra-estrutura tecnológica e organizacional de suporte ao bioempreendedorismo e de aprimoramento da relação universidade/indústria (centros de comercialização, incubadoras, parques e pólos tecnológicos);

- incentivo à formação de redes de cooperação científico-tecnológicas e à maior interação e consolidação de uma identidade sociocultural, o chamado "capital social"; 
- disponibilização de recursos e investimento de venture capital em suas modalidades;

- adoção de sólidas políticas de desenvolvimento para o setor, pela concessão de créditos fiscais e subsídios e através de programas públicos de fomento.

Até agora, o Brasil se mantém numa posição aquém desses dois modelos, tendo em vista seu mal ajustado quadro regulatório de biotecnologia e as raras ações de política de apoio industrial que sejam consistentes, pelo menos em comparação com o cenário internacional, onde existem políticas efetivamente indutoras à concessão de subsídios, ao investimento e à adoção de vantagens comerciais e fiscais para a atividade bioindustrial (JUDICE, 2004).

Diante dessa realidade, este artigo procura discutir a possibilidade de se potencializar alguns resultados positivos observados em recente pesquisa sobre o desenvolvimento bioindustrial em Minas Gerais, no período 2001-04. Para um melhor aproveitamento de elementos que podem proporcionar vantagem competitiva ao Brasil, são feitas algumas sugestões de políticas de desenvolvimento, com o intuito de contribuir para que o país possa de fato competir com os principais centros internacionais na histórica disputa da bioindústria rumo a 2020.

\section{Percurso metodológico}

O ponto de partida da atualização dos dados sobre a biotecnologia em Minas Gerais em 2004 é o diretório de empresas de 2001 mantido pela Fundação Biominas, que identifica 304 bioempreendimentos no Brasil, 89 deles naquele estado, os quais representavam $29 \%$ das empresas então existentes no país. Na identificação das empresas em 2001, biotecnologia foi considerada como a "a aplicação de diversas tecnologias 'habilitadoras', envolvendo organismos vivos, células ou moléculas para a geração de produtos e serviços" (BIOMINAS, 2001, p.7).

A pesquisa de atualização de bioempreedimentos mineiros em 2004 (JUDICE e SOARES, 2004) eliminou alguns dos segmentos de mercado considerados no estudo de 2001. Esse procedimento foi adotado para se ter uma melhor compatibilidade e comparabilidade internacional, tendo em vista estudos recentes sobre biotecnologia no Canadá, em 2001, e nos EUA, em 2002. Assim foram eliminadas 26 empresas - entre fornecedoras de equipamentos, empresas públicas e de consultoria - sobreviventes de processos de fusão e aquisição (envolvendo nove empresas, das quais restaram quatro no mercado), de mudança de setor (do farmacêutico para o de cosméticos), transferência de cidade (de Belo Horizonte para São Paulo), de fechamento e de períodos de "hibernação".

Ao final da atualização do diretório em 2004, chegou-se a um total de 83 empresas, 75 das quais efetivamente operantes no momento da pesquisa. Desse conjunto, oito empresas em fase pré-operacional foram desconsideradas, selecionando-se 42 empresas (56\%) investigadas mais detalhadamente através de questionário eletrônico dirigido a diretores e gerentes de pesquisa e desenvolvimento. Diversos aspectos foram abordados no estudo, mas em termos deste artigo, dois conjuntos de variáveis foram consideradas:

- a capacidade de inovação tecnológica, observada através de três variáveis:

- número de patentes obtidas e solicitadas no período;

- análise de conteúdo das agendas de pesquisa e desenvolvimento e de produtos,

- presença de investimento de venture capital.

- os indicadores de criação de competências para a internacionalização, cujas três principais variáveis observadas foram: ${ }^{4}$

\footnotetext{
${ }^{4}$ Foram também coletadas outras variáveis, que não serão tratadas aqui, como a "criação de bases de internacionalização" (escritórios externos) e as "relações de colaboração internacional".
} 
- a realização e/ou projeções de exportações;

- a busca de certificações e registros internacionais de qualidade e atualização de práticas internacionais de produção e fabricação;

- o desenvolvimento de técnicas avançadas de gestão.

As respostas obtidas junto às 42 empresas onde foram aplicados os questionários foram tabuladas em uma matriz de análise. Os resultados são apresentados na próxima seção, após breve caracterização desse conjunto de organizações e amostra e avaliação de correspondências e compatibilidades.

\section{Resultados}

\subsection{Caracterização das empresas de biotecnologia em M inas Gerais - distribuição geográfica, conforme os segmentos de mercado}

Das empresas em operação em Minas Gerais, 52 delas (69\%) estão localizadas na Grande Belo Horizonte incluindo cidades vizinhas num raio de $100 \mathrm{~km}$ - e $23(31 \%)$, no interior do estado. A Tabela 1 mostra a distribuição percentual dessas empresas por cidades e segmentos de mercado, mostrando que Belo Horizonte, Viçosa e o Triângulo Mineiro são as áreas de maior concentração de empresas. A Tabela 1 evidencia ainda que $56 \%$ das empresas de biotecnologia daquele estado estão concentradas no segmento de saúde humana, totalizando 42 empresas nessa área. Em segundo lugar está o segmento de saúde animal, com cerca de $15 \%$ das empresas. Agronegócios e meio ambiente aparecem em terceiro e quarto lugares, com $12 \%$ e $9 \%$ do total de empresas em funcionamento, respectivamente.

\section{Tabela 1}

Distribuição geográfica e por segmento de mercado

Empresas operantes em biotecnologia (Minas Gerais, 2004)

\begin{tabular}{|c|c|c|c|c|c|c|}
\hline Cidade/segmento de mercado & $\begin{array}{c}\text { BH } \\
\mathbf{N}=52 \\
(69 \%)\end{array}$ & $\begin{array}{c}\text { Viçosa } \\
\mathbf{N}=\mathbf{8} \\
(\mathbf{1 1 \%})\end{array}$ & $\begin{array}{c}\text { Triângulo* } \\
\text { N=6 } \\
(8 \%)\end{array}$ & $\begin{array}{c}\mathrm{JF} \\
\mathrm{N}=3 \\
(4 \%)\end{array}$ & $\begin{array}{c}\text { Outras** } \\
\mathrm{N}=6 \\
(8 \%)\end{array}$ & $\begin{array}{c}\text { TOTAL } \\
\text { N=75 } \\
(\mathbf{1 0 0 \%})\end{array}$ \\
\hline Saúde humana & 37 & - & - & 2 & 3 & 42 \\
\hline Saúde animal & 8 & 1 & 2 & - & - & 11 \\
\hline Agronegócios & 1 & 4 & 3 & - & 1 & 9 \\
\hline Meio ambiente & 3 & 1 & 1 & - & 2 & 7 \\
\hline Biologia molecular & 2 & 2 & - & - & - & 4 \\
\hline Outros *** & 1 & - & - & 1 & - & 2 \\
\hline TOTAL & 52 & 8 & 6 & 3 & 6 & 75 \\
\hline
\end{tabular}

Fonte: Pesquisa empírica realizada, 2004.

Nota: * Uberlândia e Uberaba,** Montes Claros, Varginha, Andradas, Lavras, *** Outros segmentos (química fina).

\subsection{Caracterização da amostra}

A análise da evolução da bioindústria mineira no período 2001-04 foi baseada na amostra das 42 empresas que responderam ao questionário. A correspondência entre essa amostra e o conjunto das empresas quanto à distribuição pelo estado e por segmentos de mercado é apresentada nas Figuras 1 e 2, respectivamente: 
Figura 1

Empresas de biotecnologia de Minas Gerais por segmento de mercado em 2004

Conjunto das empresas versus amostra

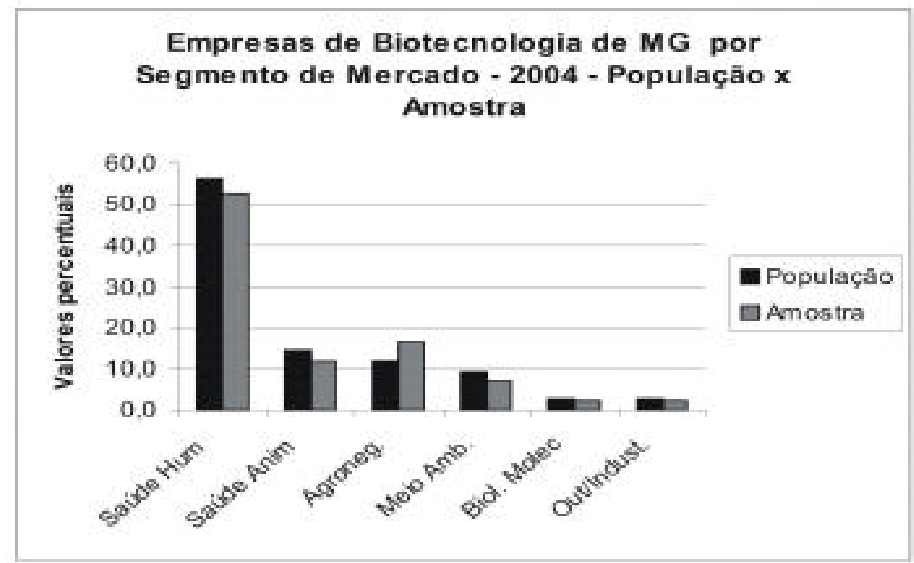

Fonte: Pesquisa empírica realizada, 2004.

Podemos observar que a distribuição geográfica e por segmentos é compatível com o verificado no conjunto das empresas, ocorrendo dois casos de "super" representação: o segmento empresarial de agronegócios e a cidade de Viçosa; uma coincidência natural, porque nessa região o agronegócio é a atividade predominante.

\section{Figura 2}

Empresas de biotecnologia em Minas Gerais em 2004

Distribuição geográfica - conjunto das empresas versus amostra (n)

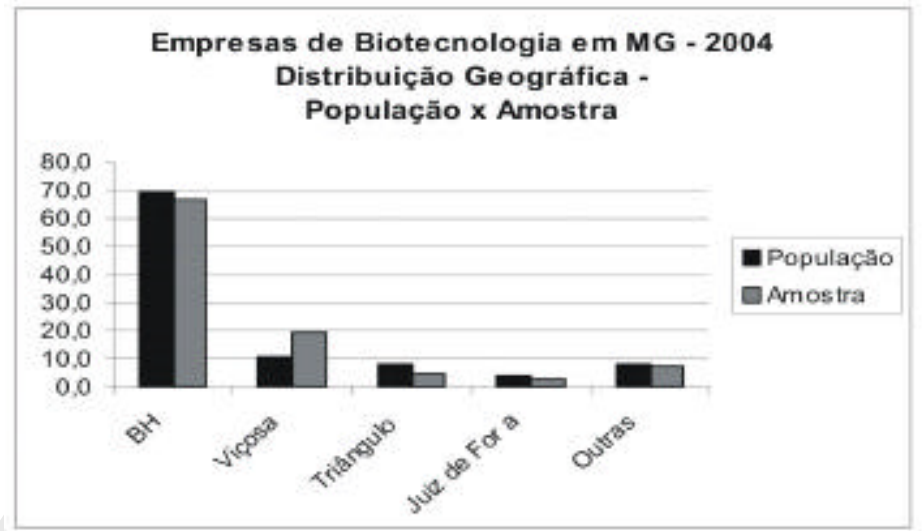

Fonte: Pesquisa empírica realizada, 2004.

\subsection{Inovação: surgimento de novas empresas com maior densidade tecnológica e potencial inovador, presença de venture capital}

Uma das constatações de maior impacto na pesquisa de atualização de informações de biotecnologia em Minas Gerais em 2004 é o surgimento de novas empresas com maior densidade tecnológica e potencial de inovação em mercados globais. Isso é indicado pelo perfil, pela orientação dos produtos e agendas de pesquisa e desenvolvimento das novas empresas emergentes, assim como pela presença mais relevante de capital de risco em 2004 em comparação ao verificado nas pesquisas de 2001 (BIOMINAS, 2001; FAJNZYLBER, 2001).

As novas empresas atuam em áreas como: 
- validação, aplicação e desenvolvimento de protocolos clínicos para drogas e protocolos relacionados ao câncer;

- novos tratamentos e produtos para prevenção e combate ao câncer;

- pesquisa e desenvolvimento relacionados a cirurgias cardíacas;

- seleção genética animal assistida por marcadores especialmente voltados para produtividade;

- exames de avaliação física de atletas assistidos por marcadores;

- serviços de biologia molecular e identificação genética de uso forense.

A maior densidade tecnológica ou maior conteúdo inovador também podem ser evidenciados no crescimento do número de patentes em relação a pesquisas anteriores. Para o conjunto de 42 empresas observadas neste estudo, foram associadas 53 patentes ( 20 concedidas e 23 solicitadas), representando uma média de 1,3 patentes por empresa, sendo $13 \%$ dessas patentes, internacionais. O número de patentes é relativamente baixo, comparado com estatísticas internacionais em biotecnologia, tanto que alguns autores questionam a capacidade de inovação desse segmento em Minas Gerais (SILVA, 2004). O estudo nacional de 2001 indicou 47 patentes (21 concedidas e 26 em processo) numa amostra de 50 empresas, com uma média de 0,94 patentes por empresa, sendo $28 \%$ dessas patentes internacionais.

Esse resultado é coerente com o que os bioempresários mineiros constaram quanto à capacidade inovadora de seus produtos nos diferentes mercados, apresentada na Tabela 2. Embora nitidamente voltados para o mercado externo, conforme já observado, esses empresários ainda se sentem relativamente menos inovadores no contexto internacional (33\%) do que no nacional (52\%).

\section{Tabela 2}

Capacidade inovadora

percepção do bioempresário $(n=42) P$

\begin{tabular}{ll}
\hline PRODUTOS / SERVIÇOS SÃO & SIM \\
\hline Inovadores no Brasil? & $52 \%$ \\
\hline Inovadores no setor? & $50 \%$ \\
\hline Inovadores internacionalmente? & $33 \%$ \\
\hline Inovadores na embalagem? & $24 \%$ \\
\hline Inovadores no design? & $21 \%$ \\
\hline
\end{tabular}

Fonte: Pesquisa - 2004.

Apesar do caráter subjetivo das respostas, a Tabela 2 serve como um indicador fundamental da mentalidade dos empresários, refletindo a maneira como constroem a auto-imagem, o que deverá ser melhor interpretado e coletivamente trabalhado para a consolidação do setor.

Uma novidade da pesquisa de 2004 foi o crescimento de investimento de venture capital, que ocorreu em $24 \%$ das empresas estudas, sendo o valor médio do investimento por empresa de $\mathrm{R} \$ 943.000$. Esse resultado contrasta com o obtido em 2001, quando apenas 7,5\% das empresas estudadas foram capitalizadas por investidores venture, nenhuma delas em Minas Gerais (BIOMINAS, 2001). Entretanto, deve-se observar que embora em crescimento, a captação de venture capital ainda é ínfima se comparada aos padrões internacionais, conforme pôde ser evidenciado nos estudos de caso internacionais pesquisados.

\subsection{Criação de competências para a internacionalização}

Nas 42 empresas pesquisadas, foram observadas as seguintes variáveis:

- exportações (primeira etapa do processo de internacionalização, conforme visto anteriormente);

- certificações e registros internacionalmente válidos; e 
- técnicas avançadas de gestão de empresas.

\subsubsection{Exportações como processo de internacionalização}

Em relação a exportações efetivamente realizadas e a projeções futuras, nota-se que o comportamento dos empresários do setor de biotecnologia em Minas Gerais vem mudando significativamente, distanciando-se de sua postura convencional, focada no mercado interno. Assim, a tradicional ausência ou baixa orientação externa - frequientemente observadas na literatura sobre internacionalização e competitividade no Brasil (OCDE, 2001; FURTADO, 2004) e também nas empresas de biotecnologia mineiras, em estudos anteriores (BIOMINAS, 2001; FAJNZYLBER, 2001) - tem se modificado, conforme mostra a recente pesquisa.

Com exceção de raras empresas ${ }^{5}$ pioneiras no processo de internacionalização, até recentemente a biotecnologia brasileira tinha em relação ao mercado, uma postura de se contentar com a "substituição de importações". Entretanto, a pesquisa mais recente mostra que uma significativa mudança está em curso. Alguns exemplos dessa mudança:

- $83 \%$ das empresas pesquisadas já exportam ou pretendem exportar para todas as regiões do mundo; enquanto no estudo anterior sobre a bioindústria mineira esse percentual era de 44\% (FAJNZYLBER, 2001);

- $40 \%$ efetivamente exportaram em 2004, enquanto em 2001, estudo nacional (empresas de todo o Brasil) identificava 28\% de exportações efetivas (BIOMINAS, 2001);

- $28 \%$ das empresas mineiras exportaram em 2004 para diversos países da América Latina (fora do Mercosul) e $62 \%$ pretendem exportar para essa região nos próximos anos;

- 14\% exportam para o Mercosul e 48\% pretendem exportar para esse bloco econômico;

- $10 \%$ exportam para os EUA e $29 \%$ têm intenção de fazê-lo num futuro próximo Na pesquisa nacional realizada em 2001, apenas $2 \%$ das empresas tinham planos de exportar para os EUA e os países-alvo para futuras exportações eram, predominantemente, Argentina, Uruguai, Paraguai, Venezuela, Colômbia, Peru, além dos países da América Central (BIOMINAS, 2001);

- 7\% exportam para a União Européia e $21 \%$ pretendem fazê-lo num futuro próximo;

- $11 \%$ exportam para a África e $26 \%$ projetam exportações futuras para essa região;

- 5\% exportam para a Ásia e 19\% têm planos de exportar para essa região (um achado de pesquisa raro, pois não há registro de qualquer orientação para essa região em nenhuma das pesquisas anteriores).

Esses resultados indicam avanço no primeiro estágio do processo de internacionalização; ou seja, o incremento das exportações, a busca de quaisquer mercados acessíveis, independentemente de curvas de aprendizado exportador e tradições geográficas e culturais já previamente existentes nas empresas.

\subsubsection{Certificações e registros com validade internacional}

Quanto às certificações e registros com validade internacional requeridos para a comercialização de produtos bioindustriais - relativos à qualidade e à salubridade dos produtos, assim como às práticas internacionais de gestão - foram consideradas as "boas práticas de manufatura" (BPM), a certificação ISO 9000 e/ou outras certificações semelhantes, além da obtenção de marca CE (Conformité Européene ${ }^{6}$ ) na Europa, da aprovação da Food and Drug Administration (FDA) nos EUA e do registro de produtos.

Foi observada uma maior procura por certificações e registros internacionais de qualidade, bem como por registros de aprovação para comercialização, em comparação com o verificado no Estudo do Setor de Diagnósticos

\footnotetext{
${ }^{5}$ A Biobras iniciou o processo de internacionalização já em seus primórdios, nos anos 1970/80 (JUDICE e BAÊTA, 2005).

${ }^{6}$ Conformidade Européia, marca de qualidade requerida para produtos comercializados na União Européia.
} 
de Minas Gerais feito em 2000 (BIOMINAS, 2000). Também foi notado um aumento no número de registros de produtos no exterior, praticamente inexistentes em 2001. Os principais destaques são:

- $64 \%$ das empresas da amostra estudada empregam "boas práticas de fabricação" (BPF) e $48 \%$ têm certificação ISO 9000. Em 2000, apenas $26 \%$ das empresas mineiras estudadas tinham esse tipo de certificação (BIOMINAS, 2000);

- é intensa a busca de outras formas de inserção no mercado internacional, através do registro de produtos. A Tabela 3 mostra como se distribuem os registros obtidos pelas respostas válidas na amostra e como se distribuiriam por toda a amostra.

Tabela 3

Registro de produtos de biotecnologia

Minas Gerais 2004

\begin{tabular}{lcc}
\hline Países/regiões de registro & Total & $\begin{array}{c}\text { Média de registros por } \\
\text { empresa }(\mathbf{n}=\mathbf{4 2})\end{array}$ \\
\hline Brasil* & 4.491 & 106,9 \\
\hline EUA & 55 & 1,3 \\
\hline União Européia & 604 & 14,38 \\
\hline América Latina & 131 & 3,1 \\
\hline \hline
\end{tabular}

Fonte: Pesquisa 2004.

Nota: * O total geral de registros obtidos é 4.491 (os registros obtidos em outros países também o foram no Brasil).

\subsubsection{Técnicas avançadas de gestão de empresas}

A utilização dessas técnicas inclui itens como planejamento estratégico, mudanças organizacionais (modelos de negócio, administração e gestão), utilização de sistemas de integração de gestão e controle de informações tecnologias de informação e comunicação (TICs) e sistemas integrados de gestão (ERPs) -, gestão de conhecimentos $(\mathrm{KM})$, gestão de cadeias de supridores (SCM) e relação com os clientes (CRM).

Três técnicas de gestão avançada de empresas se destacam pela freqüência cada vez maior com que ocorrem em empresas de biotecnologia de Minas Gerais, merecendo atenção por sua relação indireta com mecanismos de adaptação ao mercado mundial:

- tecnologias avançadas de gestão;

- inovações em marketing e comercialização e

- preocupação com treinamento sistemático e gestão do conhecimento.

\subsubsection{Tecnologias avançadas de gestão}

Aqui, foram consideradas reorganizações empresariais como: planejamento estratégico, mudanças em modelos de negócio, na gestão e administrativas. Um terço das empresas pesquisadas (33\%) vem promovendo essas reorganizações que permitem maior capacidade de adaptação às transformações do mercado, além de capacidade de internacionalização.

Em termos de utilização de tecnologias de informação e comunicação (TICs), destacam-se a preocupação com a gestão de relações com o cliente (CRM - em 19\% dos casos) e o uso ainda não muito difundido (14\% dos casos) de Business Intelligence (BI) e de sistemas integrados de gestão (ERPs).

\subsubsection{Inovações em marketing}

A comercialização e marketing de produtos têm sido apontados como uma das áreas em que as empresas brasileiras apresentam grande deficiência, quando se trata de competir no mercado internacional (FURTADO, 2004). Nesse sentido, os produtos de biotecnologia estão sujeitos a estrita regulamentação, rígidos padrões de 
qualidade, à certificação, exigência de regularidade no abastecimento e de comunicação com os mercadoschave, além de demandarem instrumentos adequados de marketing e de fortalecimento das marcas.

As empresas pesquisadas parecem estar nessa encruzilhada; tendo sido registrado que $41 \%$ delas tem buscado inovações no marketing e $29 \%$, inovações na comercialização. Esses percentuais parecem indicar conexões entre marketing e comercialização e processos de competição internacional, além de revelarem a necessidade de um especial cuidado com áreas de gestão tradicionalmente negligenciadas pelas empresas de biotecnologia no Brasil. Tais percentuais também podem indicar um fortalecimento da imagem e da competitividade das empresas, tanto no âmbito nacional quanto internacional.

\subsubsection{Treinamento sistemático e gestão do conhecimento}

Das empresas pesquisadas, $60 \%$ realizam treinamento sistemático com seus colaboradores internos e $48 \%$ se preocupam em realizar a gestão do conhecimento, elemento estratégico para a ampliação e aprofundamento de processos de inovação e de implantação de políticas de qualidade. A utilização dessas técnicas de gestão indica preocupação com qualificação permanente e competitividade, essenciais aos processos de internacionalização (OLIVEIRA JUNIOR e CYRINO, 2002).

\section{Conclusões e proposições para o desenvolvimento bioindustrial futuro}

A pesquisa realizada mostra aspectos positivos na evolução e no posicionamento da bioindústria de Minas Gerais em 2004, no que diz respeito ao potencial de desenvolvimento de produtos inovadores e à formação de competências para a internacionalização. Embora o setor empresarial não tenha tido um crescimento líquido positivo no período, novas empresas têm se apresentando no mercado. Essas empresas vêm atuando em áreas com perspectivas de inovação e que oferecem produtos de aceitação mundial, como :

- validação, aplicação e desenvolvimento de protocolos clínicos para drogas e protocolos relacionados a câncer;

- novos tratamentos e produtos para o combate e prevenção ao câncer;

- pesquisa e desenvolvimento relacionados a cirurgias cardíacas;

- seleção genética animal assistida por marcadores especialmente voltados para a produtividade;

- exames de avaliação de condicionamento físico de atletas assistidos por marcadores; e

- serviços de biologia molecular e identificação genética de uso forense.

Os indicadores de sofisticação tecnológica e capacidade de inovação são encontrados no perfil, na orientação dos produtos e nas agendas de pesquisa e desenvolvimento. São igualmente verificados pelo crescimento do número de patentes em relação a pesquisas anteriores, com uma média de 1,3 patentes por empresa, em contraposição à média de 0,94 patentes por empresa, verificada na pesquisa de 2001 .

Quanto à internacionalização, foram identificados três estágios fundamentais para o desenvolvimento das empresas, a médio e longo prazos:

- crescimento de exportações efetivas e potenciais, com mudança significativa de comportamento, visando a atuação em "todos e quaisquer mercados mundiais";

- implementação de condições e requerimentos necessários à colocação de produtos em "todos e quaisquer mercados mundiais", evidenciada pelos avanços em registros nacional e internacional de produtos, certificação de qualidade e atendimento aos padrões internacionais de "boas práticas de fabricação" (BPF); e

- difusão e implantação de técnicas avançadas de gestão e planejamento empresarial, com adoção de best management practices (uso de tecnologias de informação e comunicação, política sistemática de treinamentos, gestão do conhecimento e novos modelos de comercialização e marketing). 
Visto que os resultados aqui analisados indicam oportunidades promissoras, ainda não plenamente exploradas, mais adiante são sugeridos instrumentos de política pública potencializadores do desenvolvimento e capazes de promover e da suporte, no âmbito local, à bioindústria, visando a sua maior inovação tecnológica e inserção internacional. A expectativa é contribuir para que tais oportunidades sejam efetivamente aproveitadas não só pelo APL de biotecnologia de Belo Horizonte, mas também por outras empresas, não só em Minas Gerais como em todo o Brasil.

\subsection{Políticas de desenvolvimento bioindustrial - padrões internacionais}

A literatura sobre as experiências internacionais de desenvolvimento da bioindústria fala de modelos "fortemente" e "fracamente" indutores dessa indústria, num contexto em que o Brasil se coloca na posição de um país que praticamente não dispõe de políticas públicas indutoras. Além disso, no ambiente internacional, é verificado o recurso a diversos instrumentos políticos fundamentais para o fortalecimento bioindustrial, como:

- apoio e indução aos arranjos produtivos locais (APLs) ou clusters;

- implantação e apoio financeiro à infra-estrutura tecnológica e organizacional de suporte;

- estímulo à formação de redes de cooperação científico-tecnológicas e de interação e identidade sociocultural;

- disponibilidade de venture capital;

- criação de políticas fortemente indutoras e fornecedoras de créditos, subsídios e apoio governamental.

No Brasil, recentemente, têm aparecido indícios de algum apoio governamental mais explícito a políticas de aglomeração (APLs e clusters), através de agências públicas como o BNDES e Finep, entre outras. Adotado como política industrial no período recente, o programa de APLs apóia atividades de organização de informações e de promoção bioindustrial em arranjos produtivos brasileiros tradicionais e tecnológicos com potencial exportador. No APL de biotecnologia de Belo Horizonte, iniciado em 2004, abre-se a possibilidade de realização de diversos estudos e de aprofundar conhecimentos sobre o panorama nacional e internacional, de realizar um planejamento estratégico do setor em Belo Horizonte, de diversas iniciativas de ligação e integração com a universidade, além de programas de capacitação gerencial e tecnológica. Entretanto, sabe-se que essa proposta de apoio nacional aos APLs é um tanto precária, pelas resistências que sofre por causa das políticas macroeconômicas adotadas no país e de eventuais desdobramentos de programas de crescimento econômico, se considerarmos a realidade política brasileira, em um ano eleitoral como 2006. Portanto, é previsível alguma descontinuidade.

Por outro lado, existe indicador de uma política nacional mais proativa e alinhada com tendências internacionais. Trata-se do fato da nova política industrial passar, em 2004, a incluir a biotecnologia como uma das "áreas portadoras de futuro", adotando, entre as medidas importantes para ao desenvolvimento dos setores bioindustriais, a implantação do Fórum de Competitividade e a compra de equipamentos e contratação de bolsistas para o Centro de Biotecnologia da Amazônia (CBA). Em fármacos e medicamentos, além das discussões sobre as normas a serem adotadas no âmbito das "boas práticas de fabricação" (BPF) e rastreabilidade para fármacos, podem ser mencionados a criação do Profarma, linha do BNDES para financiamento do setor e a criação da Empresa Brasileira de Hemoderivados e Biotecnologia (Hemobras), aprovada em novembro de 2004 pelo Congresso Nacional (BALANÇO, 2005).

\subsection{Proposições de desenvolvimento bioindustrial em Minas Gerais e no Brasil - organização de informações para}

\section{a ação}

Este artigo é concluído sugerindo-se alguns instrumentos de organização setorial nacional e estadual e de ferramentas para a criação de referenciais externos que sirvam de parâmetro para ações de desenvolvimento bioindustrial no Brasil; especialmente, em Minas Gerais: 
- constituição e organização de sistemas dinâmicos de monitoramento e atualização de informações sobre o estado da arte e desenvolvimentos de políticas e investimentos públicos e privados nos níveis internacional e nacional;

- que se empreendam esforços para a quantificação de recursos internacionais e nacionais investidos no setor (benchmarking);

- estruturação de sistemas de informação e conhecimento (bases de dados) de apoio ao bionegócio, acessáveis pela internet e facilmente disponíveis;

- organização de sistemas de divulgação de informações e conscientização do público sobre a evolução, os avanços internacionais e a importância dessa indústria para o desenvolvimento;

- criação de agências e observatórios de tendências tecnológicas e formação de redes nacionais/internacionais de cooperação em inovação.

As propostas aqui apresentadas podem contribuir para um maior conhecimento e uma melhor instrumentalização de ações de concentrações e APLs bioindustriais brasileiros, assim como para a formação e o esclarecimento da opinião pública sobre o papel da bioindústria no contexto brasileiro. Todavia, trata-se de uma primeira equiparação com o padrão das práticas internacionais no setor. É preciso que instrumentos mais fortemente indutores e proativos de intervenção política pública - a exemplo do modelo-1 internacional sejam mobilizados e praticados nacional e regionalmente, se de fato há intenção de se desenvolver a bioindústria no Brasil nos próximos 15 ou 20 anos. 


\section{Referências bibliográficas}

AUDRETSCH, D. B. The role of small firms in the U.S. biotechnology clusters. Small Business Economics, Dordrecht, The Netherlands, v.17, n.1/ 2, p.3-16, Aug./Sept. 2001.

BADIN, C. Inovação na gestão ao mercado externo. RAE Eletrônica, v.2, n.1, p.1-9, 2003. Disponível em: <www.rae.com.br/eletronica>.

BALANÇO da Política Industrial. Jornal da Ciência, 4 mar. 2005. Disponível em: <http://www.jornaldaciencia.org.br/Detalhe.jsp?id=25966>. Acesso em: 04 mar. 2005.

BIOMINAS. Estudo de setor de diagnósticos em Minas Gerais. Relatório de pesquisa realizada para o Instituto de Desenvolvimento Industrial de Minas Gerais (Indi), Departamento de Relações Internacionais, Eurocentro de Belo Horizonte e Programa AL-Invest da União Européia. Belo Horizonte, 2000.

Parque Nacional de Empresas de Biotecnologia. Study prepared for the Ministry of Science \& Technology, MCT. Final report presented in Dec. 2001. Disponível em: <http://www.mct.gov.br/Temas/biotec/estudos_biotec_parque.htm>. Acesso em: 15 mar. 2004.

CASPER, S.; KARAM ANOS, A. Commercializing science in Europe: the Cambridge biotechnology cluster. European Planning Studies, Glasgow, UK, v.11 n.7, p.805-822, Oct. 2003.

CASSIOLATO, J. E.; LASTRES, H. M. M. Local systems of innovation in Mercosur countries. Industry and Innovation, Copenhagen, v.7, n.1, p.33-54, Jun. 2000.

G Glossário de arranjos e sistemas produtivos e inovativos locais. Rede de pesquisa em sistemas produtivos e inovativos locais. Rio de Janeiro: UFRJ; Sebrae, nov. 2003. Disponível em: <www.ie.ufrj.br/redesist>. Acesso em: 20 abr. 2004.

COOKE, P. Biotechnology clusters in the UK: lessons from localisation in the commercialisation of science. Small Business Economics, Dordrecht, The Netherlands, v.17, n.1/2, p.43-60, Aug./Sept. 2001.

The evolution of biotechnology in the three continents: Schumpeterian or Penrosian? European Planning Studies, Glasgow, UK, v.1̄ n.

DE VOL, R. et al. America's biotech and life science clusters. San Diego's position and economic contributions. Technical Report, Milken Institute, Santa Monica, CA, 2004.

FAJNZYLBER, P. Fatores de competitividade e barreiras ao crescimento no pólo de biotecnologia de Belo Horizonte. Estudo preparado para a Cepal. Departamento de Economia e Cedeplar - UFMG, Belo Horizonte, 2001.

FEDERAÇÃO DAS INDÚSTRIAS DO ESTADO DE M INAS GERAIS (FIEM G). Cresce Minas: um projeto brasileiro. Belo Horizonte, 2000.

FELDMAN, M. P.; FRANCIS, J. L. Fortune favours the prepared region: the case of entrepreneurship and the capitol region biotechnology cluster. European Planning Studies, Glasgow, UK, v.11, n.7, p.765-788, Oct. 2003.

; RONZIO, C. R. Closing the innovative loop: moving from the laboratory to the shop floor in biotechnology manufacturing. Entrepreneurship and Regional Development, Växjö, Sweden, v.13, n.1, p.1-16, Jan. 2001.

FELLER, G. Biotech industry grows in India. Research Technology Management, Arlington, VA, p.46, Jul./Aug. 2003.

FLEURY, A.; FLEURY, M. T. L. Por uma política industrial desenhada a partir do tecido industrial. In: FLEURY, M. T. L.; FLEURY, A. Política industrial. São Paulo: FEA-USP. v.1. (Biblioteca Valor Publifolha, 2004. p.79-107.).

FURTADO, J. Quatro eixos para a política industrial. In: FLEURY, M. T. L.; FLEURY, A. Política industrial. São Paulo: FEA-USP. v. 1. (Biblioteca Valor Publifolha, 2004. p.47-78.).

JUDICE, V. M. M. Sete experiências internacionais de desenvolvimento da bioindústria. Relatório de pesquisa. Instituto Euvaldo Lodi (IEL- MG), Belo Horizonte, 2004.

; BAÊTA, A. M. C., 2002. Clusters em bioindústria e biotecnologia em Minas Gerais. Habitats construídos de inovação, competitividade e desenvolvimento regional. Gestão \& Tecnologia, Pedro Leopoldo, v.1, n.1, p.155-170, nov. 2002.

Gestão de inovação e fatores de competitividade na bioindústria - desafios à evolução do cluster de biotecnologia em Bèlo Horizonte (M G). In: X seminario latino iberoamericano de gestión tecnológica Altec 2003. Conocimiento, innovación y competitividad: los desafíos de la globalización, Ciudad de México, DF, 22, 23 y 24 de octubre 2003.

; SOARES, E. E. Diagnóstico biotecnologia em Minas Gerais 2004. Desafios rumo a 2010: inovação, internacionalização e governança do setor. Relatório de pesquisa. Instituto Euvaldo Lodi (IEL-MG), Belo Horizonte, 2004.

KAISER, R. Multi-level science policy and regional innovation: the case of the Munich cluster for pharmaceutical biotechnology. European Planning Studies, Glasgow, UK, v.11, n.7, p.841-857, Oct. 2003.

KANDIAH, P. Hub to boost biotech research. Managing Intellectual Property, London, UK, issue 34, p.71-72, Nov. 2003.

KAUFM ANN, D. et al. The role of location for biotechnology firms in Israel. European Planning Studies, Glasgow, UK, v.11, n.7, p.823-840, Oct. 2003. 
LASTRES, H. M. M.; CASSIOLATO, J. E.; M ACIEL, M. L. Pequena empresa. Cooperação e desenvolvimento local. Rio de Janeiro: Relume Dumará; UFRJ- Instituto de Economia, 2003.

LEMOS, M. B. A construção de redes locais de inovação sob condições periféricas: o caso da biotecnologia na aglomeração de Belo Horizonte. Projeto Globalização e Inovação Localizada: Experiências e Sistemas Locais no Âmbito do Mercosul e Proposições de Políticas de C\&T. UFRJ-Instituto de Economia, 2000. Disponível em: <www.ie.ufrj.br/redesist>. Acesso em: 02 abr. 2004.

MARCOLIN, N. Da botica à sala de aula. Primeira escola de farmácia foi criada há 165 anos em Outro Preto. Pesquisa Ciência e Tecnologia no Brasil, Fapesp, São Paulo, p.8- 9, mar. 2004.

NIOSI, J.; BAS, T. G. Biotechnology megacentres: Montreal and Toronto regional systems of innovation. European Planning Studies, Glasgow, UK, v.11 n.7, p.789-804, Oct. 2003.

OCDE. Estudos Econômicos da OCDE - Brasil, 2000-2001. Rio de Janeiro: FGV, 2001.

OLIVEIRA JUNIOR, M. M.; CYRINO, A. B. Global players - pesquisa sobre a internacionalização da empresa brasileira. Nova Lima: Fundação Dom Cabral, 2002. Disponível em: <http://www.fdc.org.br/conteudo.asp?cod=246>. Acesso em: maio 2004.

OLIVER, A. L. Strategic alliances and the learning life-cycle of biotechnology firms. Organization Studies, Berlin, v.22, n.3. p.467-489, 2001.

PEREZ, C. The life and times of a technology. Recurring phases of each great surge. Image presented in "Coming of age, a survey on IT industry". The Economist, London UK, May, 10th, 2003.

PORTER, M. E. Clusters e competitividade. HSM Management. v.4, n.15, p.100-110, jul./ago. 1999a.

Competição. On competition. Estratégias competitivas essenciais. 6. ed. Rio de Janeiro: Campus, 1999b.

POWELL, W. W. et al. The spatial clustering of science and capital: accounting for biotech firm-venture capital relationships. Regional Studies, Cambridge, UK, v.36, n.3, p.291-306, May 2002.

SAINSBURY OF TURVILLE, D. Biotechnology clusters. Report of a team lead by Lord Sainsbury. Minister for Science - Department of Trade and Industry (DTI, UK), London, UK, 1999.

SANG, K-R. New funding for biotech sector. Asia Pacific Biotech News, Singapore, v.7 n.24, p.2-5, 2003.

SCATOLIN, F. D et al. Arranjos produtivos e dinâmica do comércio internacional. In: LASTRES, H. M. M.; CASSIOLATO, J. E.; MACIEL, M. L. Pequena empresa. Cooperação e desenvolvimento local. Rio de Janeiro: Relume Dumará; UFRJ-Instituto de Economia, 2003. p.137-152.

SILVA, E. R. A rede de inovação no cluster de biotecnologia de Belo Horizonte. Um estudo sobre rede social, ambiente local e capacitação tecnológica. Dissertação (Mestrado em Ciências Sociais) - PUC de Minas Gerais, Belo Horizonte, 2004.

SIMON, F.; KOTLER, P. A construção de biomarcas globais. Levando a biotecnologia ao mercado. São Paulo: Bookman, 2004.

SOUZA, M. C. A. F et al. Perspectivas para uma atuação competitiva das pequenas empresas no contexto atual. In: LASTRES, H. M. M.; CASSIOLATO, J. E.; MACIEL, M. L. Pequena empresa. Cooperação e desenvolvimento local. Rio de Janeiro: Relume Dumará; UFRJ-Instituto de Economia, 2003. p.225-242.

SOUZA, S. G. A. Biotecnologia em Minas Gerais: potencialidades e desafios para o desenvolvimento regional. Diamantina (MG). X Seminário sobre Economia Mineira, Centro de Desenvolvimento e Planejamento Regional (CEDEPLAR) UFMG, 2002.. Disponível em http://cedeplar.ufmg.br/seminarios/sub sem dia 2002 tex.php, acessado em 05/06/2005.

TRAORÉ, Namatié. "Biotechnology use and development survey: methodology, issues and responses". STATISTICS CANADA, Science, Innovation and Electronic Information Division, Working Papers no. 88F0006XIE - No. 006, 2003. Disponível em http://www.statcan.ca/english/research/88F0006XIE/88F0006XIE2004006.pdf acessado em 05/06/2005.

TALLMAN, S. et al. Knowledge clusters and competitive advantage. Academy of Management Review, New Orleans, LA, v.29, n.2, p.258271, 2004.

U.S. DEPARTMENT OF COM MERCE, A Survey of the use of biotechnology in U.S. industry. Technology Administration, Bureau of Industry and Security, October 2003. Disponível em http://www.technology.gov/reports/Biotechnology/CD120a 0310.pdf , acessado em 05/06/2005.

WEISENFELD, U.; REEVES, J. C.; HUNCK-MEISWINKEL, A. Technology management and collaboration profile: virtual companies and industrial platforms in the high-tech biotechnology industries. R\&D Management, Manchester, UK, v.31, n.1, p.91-100, 2001.

ZOLLO, M.; REUER, J.; SINGH, H. Interorganizational routines and performance in strategic alliances. Organization Science, Linthicum, MD, v.13, n.6, p.701-713, Nov./Dec. 2002. 